\title{
Sobre os Trilobitas da Bacia do Paraná e o Centenário da Monografia de Clarke (1913) e O Mito de Sísifo na Paleontologia Brasileira
}

\section{Trilobites on the Paraná Basin and the Centenary of Clarke Monograph (1913) and The Myth of Sisyphus in Brazilian}

Paleontology

\section{Acerca de los trilobites de la Cuenca del Paraná y el Centenario de la monografía Clarke (1913) y El mito de Sísifo en la paleontología brasileña}

Renato Pirani Ghilardi

ghilardirp@gmail.com Universidade Estadual de São Paulo

Drielli Peyerl

driellipeyerl@gmail.com Universidade Estadual de Campinas

Juliana Moraes Leme leme@usp.br Universidade de São Paulo

André Mori

andr_mori@hotmail.com Universidade de São Paulo

Resumo: Estabelece-se aqui o histórico de pesquisas desenvolvidas em trilobitas na Bacia do Paraná, Brasil. Desde o clássico trabalho monográfico de J. M. Clarke até o presente várias fases de desenvolvimento científico ocorreram, a saber: uma primeira fase de prospeç̧ão de material, uma segunda fase de estudos sistemáticos e uma terceira fase com a consolidação dos conceitos adquiridos. Adicionalmente, discutemse as espécies de trilobitas descritas para o devoniano da Bacia do Paraná, elencando as espécies válidas. Por fim, apresentam-se comentários sobre o desenvolvimento das pesquisas paleontológicas brasileiras, principalmente no que se refere ao estudo de trilobitas.

Palavras-chave: História da Paleontologia. Bacia do Paraná. Paleoinvertebrados. Trilobita. Filosofia da Ciência. Centenário Clarke 1913. 


\begin{abstract}
The history of research done with trilobites from the Basin Paraná, Brazil, is reported here. From the classic monograph by J. M. Clarke to the present, various stages of scientific development occurred, namely: the first phase of prospecting for material, a second phase of systematic studies, and a third phase for the consolidation of acquired concepts. Additionally, we discuss the species of trilobites described for the Devonian of the Paraná Basin, listing the valid species. Finally, it is explained the growth of Brazilian paleontological research, especially regarding the study of trilobites.
\end{abstract}

Keywords: History of Paleontology. Paraná Basin. Paleoinvertebrates. Trilobita. Philosophy of Science. Clarke 1913 Centenary.

Resumen: Se establece aquí la historia de la investigación desarrollada en trilobites de la Cuenca Paraná, Brasil. Desde el clásico monografía J. M. Clarke se produjeron a las presentes, diferentes etapas de desarrollo científico, a saber: una primera fase de material de exploración, una segunda fase de estudios sistemáticos y una tercera fase a la consolidación de los conceptos adquiridos. Además se discute las especies de trilobites descritas para el Devónico de la cuenca del Paraná para enumerar las especies válidas. Por último, se dice comentarios sobre el desarrollo de la investigación paleontológica de Brasil, principalmente en relación con el estudio de los trilobites.

Palabras-clave: Historia de la Paleontología. Cuenca Paraná. Paleoinvertebrados. Trilobita. Filosofía de la Ciencia. Centennial Clarke 1913.

\title{
CONTEXTUALIZAÇÃO HISTÓRICA
}

A história e o desenvolvimento da pesquisa dos trilobitas no Brasil, já discutida e trabalhada por Ghilardi \& Simões (2007), pode ser dividida em três grandes fases, que os autores separaram em relação ao escopo e à quantidade de pesquisadores envolvidos nessa temática.

A primeira fase trata das grandes expedições exploratórias que tiveram como um dos intuitos prospectar e coletar o máximo de informações paleontológicas possíveis dentre as bacias sedimentares brasileiras. Tendo como exemplo a Comissão Geológica do Brasil - CBG (1875 - 1878), que pode ser considerada "como a primeira iniciativa institucional, de abrangência nacional, no âmbito específico das ciências geológicas no Brasil" (FIGUEIRÔA, 1997, p. 150).

A segunda fase teve a duração considerável de cerca de 80 anos, que corresponde à fase de estudo de pesquisadores estrangeiros com alguma contribuição, também, de pesquisadores brasileiros em relação ao material coletado, principalmente de cunho paleontológico (GHILARDI \& SIMÕES, 2007).

Em relação à última fase, ela ocorre a partir da virada do séc. XXI, sendo caracterizada pela associação de conceitos mais amplos e distintos à sistemática usada até então. É possível observar seus reflexos ainda nos dias de hoje.

Dentre as fases citadas anteriormente, destacamos que esse trabalho se restringirá às pesquisas da segunda e da terceira fases, por meio das quais será possível demonstrar 
o embasamento científico (principalmente o taxonômico) que promoveram todas as pesquisas realizadas no fim do séc. XX e início do séc. XXI.

\section{CLARKE E A SISTEMATIZAÇÃO DOS TRILOBITAS DEVONIANOS}

Para se compreender melhor como o geólogo e paleontólogo John Mason Clarke (1857 - 1925) escreveu sua célebre monografia de 1913, há a necessidade de se recuar no tempo e contextualizar o momento histórico paleontológico de meados de 1870.

Em 1879, Orville Adelbert Derby (1851 - 1915) foi nomeado diretor da Seção Geológica e Mineralógica do Museu Nacional do Rio de Janeiro. Tal acontecimento se mostrou de suma importância para o desenrolar dos fatos, pois, naquela época, Ladislau de Souza Mello Netto (1838 - 1894), diretor do Museu Nacional, incentivou Derby a enviar para o exterior o material acumulado pela Comissão Geológica do Brasil (a partir de agora CGB) em suas prospecções. Derby tinha a consciência de que o material coletado pela CGB deveria ser estudado, descrito e catalogado dentro de um padrão de excelência. Dessa forma, pesquisadores como E. D. Cope, C. Branco , J. M. Clarke, e Richard Rathbun (1852 - 1918), receberam material fóssil da CGB para trabalho.

Mesmo com a visão pioneira de Derby, os resultados práticos de sua ação demoraram a se concretizar na forma de trabalhos monográficos, como os de J. M. Clarke (1890, 1913) e Friedrich Katzer (1903). Não obstante, essas monografias se tornaram os estudos pioneiros de trilobitas no Brasil, incluindo a Bacia do Paraná (GHILARDI \& SIMÕES, 2007). Ressalta-se, ainda, que as monografias mencionadas foram publicadas pelo Serviço Geológico e Mineralógico do Brasil, criado em 1907.

De fato, em 1890, Clarke apresentou o primeiro estudo monográfico dos invertebrados marinhos do Paleozoico do Brasil, incluindo o material das formações Ererê e Maecurú da Bacia do Amazonas (CLARKE, 1890). Fato curioso é que no apêndice dessa monografia, Clarke faz uma indicação direta de seu relacionamento científico com Derby. Conforme seu texto, na página 55, Clarke menciona "Since preparing the foregoing descriptions of Maecurú and Ereré trilobites, I have received from Prof. Derby, accompanied by a request that a description should be prepared, specimens of a trilobite obtained at Jaguarahyva, Paraná, near the southern boundary of S. Paulo". Entretanto, a descrição do material de Jaguariaíva, bem como das outras localidades do Devoniano da Bacia do Paraná, da Argentina e das Ilhas Falklands apenas ocorre em seu clássico legado: Monografia número 1 do Serviço Geológico e Mineralógico do Brasil, publicado em 1913.

Nesse trabalho, Clarke (1913) apresentou um estudo detalhado das faunas de invertebrados fósseis da Bacia do Paraná, sul do Brasil. Em particular aos trilobitas, descreve treze novas espécies (Homalonotus noticus, Homalonotus (Schizopyge) parana, Dalmanites accola, Dalmanites sp., Cryphaeus australis, Cryphaeus sp., Calmonia signifer, Calmonia signifer var. michrischia, Calmonia subseciva, Calmonia ? gonzagana, Pennaia pauliana, Proboloides cuspidatus, Proboloides pessulus). 
O autor ainda observou a natureza austral dessa fauna, concluindo que as bacias Amazonas e Paraná não estariam conectadas durante esse período. Ademais, Clarke considera a fauna de paleoinvertebrados de idade devoniana inicial. Não há dúvidas de que esse estudo hercúleo se constitui um dos mais importantes trabalhos sobre a fauna devoniana do Brasil.

Curiosa e concomitantemente, no mesmo ano de publicação da monografia de Clarke, Roman Kozlowski, com base na fauna de invertebrados coletados em Jaguariaíva, Paraná, descreve três novas espécies de trilobitas (Homalonotus sp., Acaste lombardi e Cryphaeus sp.). Como a publicação foi posterior ao trabalho de Clarke, ele não obteve a prioridade dos termos. (BOSETTI et al., 2007).

Outro trabalho que merece destaque é o de Petri (1948), que conseguiu recuperar os dados de publicação dos trabalhos de Clarke e Koslowski, demonstrando que, por uma diferença de poucos meses, o trabalho do primeiro autor teria prioridade taxonômica sobre as descrições do segundo que, em 1923, assume a não prioridade nomenclatural de seu trabalho (KOZLOWSKI, 1923).

\section{HISTÓRICO PARA OS TÁXONS VÁLIDOS DE TRILOBITAS}

A monografia de Clarke se mostrou tão fortemente embasada que por cerca de 60 anos não houve publicações relevantes quanto a alterações da sistemática do grupo de trilobitas para a Bacia do Paraná. Associado a isso, pode-se dizer que a partir da década de 1980, o trabalho nessa área foi caracterizado pelo desaparecimento dos pesquisadores vinculados a museus, comissões ou instituições científicas que trabalhassem com esses grupos, dando lugar a pesquisadores com treinamento e visões mais acadêmicas do assunto.

De fato, durante esse período, foram publicados, maiormente, séries de pequenas notificações, resumos e listas de fauna, como os trabalhos de Lange (1954) e Lange \& Petri (1967), os quais listam fósseis para o Devoniano da Bacia do Paraná alterando táxons propostos por Clarke (1913). A pesquisa em trilobitas no Brasil foi a partir de então esquecida até meados da década de 1980, quando merecem destaque os trabalhos de Maria da Glória Carvalho e Marlene Terezinha Barcellos-Popp, que recomeçam os estudos desses grupos. Carvalho, por sinal, em alguns dos seus trabalhos (CARVALHO et al., 1987; CARVALHO \& EDGECOMBE, 1991) estuda material coletado nos estados de Mato Grosso do Sul e de Goiás (flanco noroeste da Bacia do Paraná) que não foram incluídos nos estudos de Clarke, conferindo um caráter pioneiro a esses estudos, uma vez que previamente apenas Ammon (1893) e Oliveira (1937) descrevem trilobitas para essas regiões. Esses trabalhos atribuem uma fauna dominada por calmoniídeos (Metacryphaeus australis, Metacryphaeus sp., Calmonia subseciva, Calmonia cf C. signifer, Calmonia triacantha e Paracalmonia sp. ?) e homalonotídeos (Burmeisteria sp.).

Por sua vez, Popp (1985) em sua tese de doutorado, revisou os trilobitas descritos em 1913 por Clarke (com exceção de Homalonotidae), sugerindo a presença de no- 
vos gêneros e espécies. Em uma publicação derivada de seu doutoramento, Popp (1989) formaliza e valida apenas o táxon Paranacaste pontagrossensis. No entanto, Carvalho \& Edgecombe (1991) observam que Paranacaste é sinônimo de Bainella pontagrossensis, com parentes próximos na Bolívia e na África do Sul. Em um novo estudo, Popp et al. (1996), apresentam a primeira hipótese filogenética para trilobitas, em específico do gênero $\mathrm{Pa}$ racalmonia, da Bacia do Paraná, propondo novos táxons e formulando uma hipótese de filogenia para Paracalmonia.

Devem-se citar ainda outros autores (COPPER, 1977, COOPER, 1982; ELDREDGE \& ORMISTON, 1979; ELDREDGE \& BRANISA, 1980) que também mencionam trilobitas da Bacia do Paraná (por vezes alterando a sistemática do táxon), utilizando-os em trabalhos mais relacionados com aspectos paleobiogeográficos.

A maioria desses trabalhos, contudo, não têm os cuidados necessários para que os táxons propostos tenham consistência e formalidade dentro dos termos do Código de Nomenclatura Zoológica Internacional, revelando a fragilidade e a inexistência de muitas propostas.

A partir do séc. XXI, a visão sobre os trabalhos paleontológicos sofre novamente uma quebra de paradigma. Conceitos como os de tafonomia, paleossinecologia e estratigrafia de sequências são incorporados aos estudos dos trilobitas da Bacia do Paraná de forma mais premente. A visão de que uma paleontologia de alto nível poderia ser feita apenas com exemplares de gaveta de museu foi aos poucos abolida dentro do pensar paleontológico. De fato, os trabalhos começaram a tratar os trilobitas como ferramentas associadas a estudos mais abrangentes. Trabalhos como os de Ghilardi \& Simões (2000, 2001) ou como o trabalho de doutoramento de Ghilardi (2004) conseguem dar aos trilobitas do Devoniano um caráter mais prático para os estudos paleontológicos, inclusive associando previsibilidade aos padrões de preservação desse grupo aos diferentes tratos de sistema dentro da sequência sedimentar.

Soares (2007) e Simões et al. (2009) apresentaram uma revisão sistemática e tafonômica dos Homalonotiidae da Formação Ponta Grossa, indicando que a fauna de Homalonotidae da Formação Ponta Grossa não é monoespecífica, conforme anteriormente pensado, tendo a ocorrência de duas espécies, Burmesteria noticus e B. herscheli. Adicionalmente, Simões et al. (2009) observaram que os homalonotídeos da Formação Ponta Grossa não estão distribuídos aleatoriamente ao longo da Formação Ponta Grossa, ou seja, trilobites homalonotídeos são mais abundantes nas fácies de águas mais rasas, depositadas acima do nível de base de ondas de tempestade, sendo, potencialmente, importantes indicadores paleoambientais.

O primeiro registro de Metacryphaeus rotundatus em rochas devonianas, do Brasil, Formação Ponta Grossa, foi documentado por Soares et al. (2008a). M. rotundatus é uma espécie de ocorrência comum em rochas emsianas, da Formação Icla, Bolívia. Essa nova ocorrência reforçou outras evidências paleontológicas indicativas da presença de elementos cosmopolitas, da Província Andina, nas assembleias fósseis do Devoniano da Bacia do Paraná. 
O trabalho de Soares et al. (2008b) traz à tona um dos maiores problemas do estudo da sistemática dos trilobitas da Bacia do Paraná: a presença de tafotáxons. Muitas das feições diagnósticas dos elementos da fauna de trilobitas são derivadas de medições angulares ou muito subjetivas, dificultado a correta classificação. Além disso, vários gêneros e espécies de trilobitas apresentam grande similaridade morfológica entre si, e, portanto, são diagnosticadas em parte por mínimos e frágeis detalhes. A esse fato, agrava as alterações provenientes dos processos tafonômicos associados à preservação dos espécimes.

Mori \& Leme (2012) e Mori (2013), após uma extensa revisão sistemática de Calmoniídae da Formação Ponta Grossa, mostraram que o levantamento do número de espécies dessa família de trilobitas na Formação Ponta Grossa foi superestimado e, portanto, não refletia um panorama confiável da diversidade de calmoniídeos. O número de tafotáxons apontados correspondeu a praticamente metade dos nomes específicos descritos até então (MORI, 2013). Esses autores estimam que nove espécies de Calmoníídeos sejam válidas para a Formação Ponta Grossa (Figura 1 e Tabela 1).

Cabe ressaltar que a descrição do material tipo foi realizada de forma clara, objetiva e bem organizada por Clarke (1913), de modo que até hoje influencia e deve ser tomada como referência para trabalhos taxonômicos de trilobitas calmoniídeos. O material tipo analisado por Clarke se encontra na coleção paleontológica do Departamento Nacional de Produção Mineral (DNPM) e corresponde a uma das melhores e mais numerosas coleções de calmoniídeos do país.

No entanto, o trabalho de Clarke (1913) e o material tipo não estão isentos de problemas, principalmente em relação à qualidade de preservação de certos exemplares, incluindo holótipos. A presença de tafotáxons fica mais evidente quando se alia a visão tafonômica ao estudo taxonômico, fundamentado, então, na consideração de que certos caracteres diagnósticos são vulneráveis e passíveis de alteração perante os vários fatores externos aos quais os organismos originais e os fósseis estão expostos (Soares et al. 2008b; Mori \& Leme 2012; Mori, 2013). Isso demonstra que até mesmo trabalhos icônicos e de qualidade como a monografia de Clarke devem passar por revisões que se encaixam nos paradigmas atuais, sempre visando à otimização das informações e ao conhecimento que podem ser obtidos desses clássicos trabalhos. 
Figura 1 - A-G - Calmoniídeos da Formação Ponta Grossa. A - Metacryphaeus australis; holótipo (DGM31). B - Paracalmonia cuspidata; Holótipo (DGM78). C - Calmonia signifer; holótipo (DGM17). D - Pennaia pauliana; holótipo (DGM71). E - Paraphacopina polygona (GP/1E7493). F - Paranacaste pontagrossensis; holótipo (127Tr). G - Metacryphaeus rotundatus (DGM69). Fotos A-E e G adaptadas de Mori (2013). Foto F adaptada de Carvalho \& Edgecombe (2006). Escala $=5 \mathrm{~mm}$

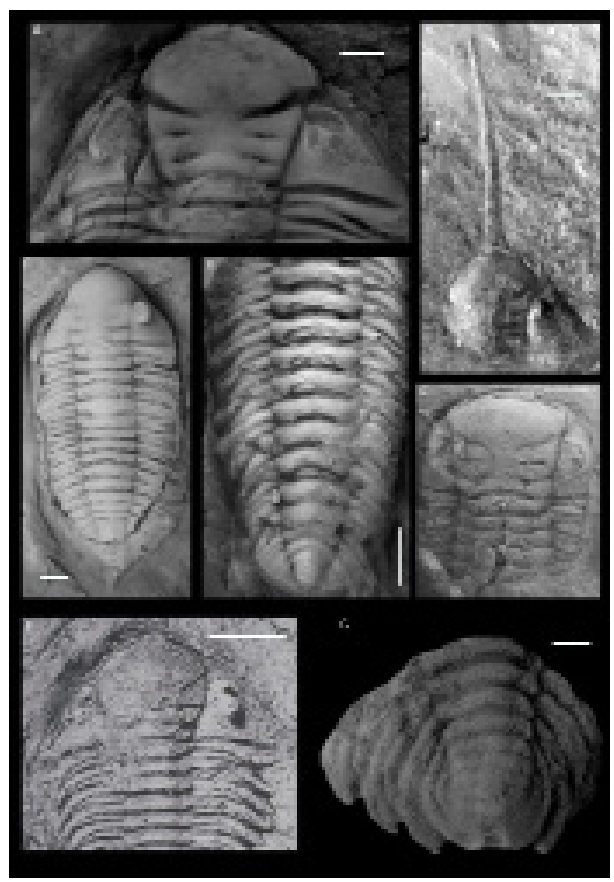

Tabela 1 - Lista de espécies de calmoniídeos descritos e válidos para a Formação Ponta Grossa, Devoniano, Bacia do Paraná, Brasil

Calmoniidae Formação Ponta Grossa

\section{Autor}

\begin{tabular}{c|c} 
Acaste lombardi & Kozlowski, 1923 \\
\hline Bainella paranaense & Carvalho \& Edgecombe, 1991 \\
\hline Calmonia signifer & Clarke, 1913 \\
\hline Metacryphaeus australis & Clarke, 1913 \\
\hline Metacryphaeus rotundatus & Kozlowski, 1923 \\
\hline Paracalmonia cuspidata & Clarke, 1913 \\
\hline Paranacaste pontagrossensis & Popp, 1989 \\
\hline Paraphacopina polygona & Mori \& Leme, 2013 \\
\hline Pennaia pauliana & Clarke, 1913
\end{tabular}




\section{CONSIDERAÇÕES FINAIS}

Indubitavelmente, o trabalho de Clarke (1913) é um marco dentro das pesquisas do devoniano da Bacia do Paraná. Suas ponderações e colocações ainda são fortemente utilizadas e são consideradas cientificamente muito bem elaboradas. Contudo, além da parte científica, sua monografia nos traz também a possibilidade de reflexões sobre uma faceta da filosofia da ciência paleontológica brasileira: por que há um intervalo tão grande de tempo sem publicações relevantes sobre a paleontologia dos trilobitas da Bacia do Paraná?

Camus (1942) elabora um ensaio chamado "Mito de Sísifo", no qual retrata, dentro da filosofia, o trabalho eterno, porém infrutífero, de as pessoas darem sentido real a sua existência. Com suas devidas proporções, esse raciocínio pode ser implementado nas ciências brasileiras, como a paleontológica. De fato, muito do que se considera "ciência" dentro dos padrões nacionais nada mais é do que um amontoado de compilações ou tentativas infrutíferas de dar sentido real àquilo que se faz.

Obviamente, é necessário que ocorram mudanças dentro do processo científico, uma fase inicial de descrição e de catalogação dos espécimes em estudo seria um primeiro passo para que haja possibilidade de ordenação e desenvolvimento das ideias. A continuidade a partir desse ponto é que não ocorre, muitas vezes devido a uma inconsistência de padronização e/ou morosidade científica.

As pesquisas realizadas no início do século XXI são, na verdade, produtos do período de adequações relacionadas ao surgimento de um novo paradigma paleontológico nacional que necessita de novos princípios, teorias, conceitos básicos e metodologias para que consiga um salto qualitativo de seus resultados em relação à clássica monografia de Clarke (1913), por exemplo.

Clarke (1913) sempre foi e será lembrado como um trabalho divisor de conhecimento para os trilobitas da Bacia do Paraná. Que seja lembrado daqui a 100 anos como parte do primeiro degrau de uma escadaria para a qual a subida faz sentido.

\section{REFERÊNCIAS}

AMMON, L. von. Devoniche Versteinerunger von Lagoinha in Mato Grosso (Brasilien): Zeitschrift der Gesellschaft für Erdkunde zur Berlin, n. 28, 1893, p. 352-366.

BOSETTI, E. P.; PEYERL, D.; HORODYSKI, R. S.; ZABINI, C. Formação Ponta Grossa: História, Fáceis e Fósseis. In: I Simpósio de Pesquisa em Ensino e História de Ciências da Terra / II Simpósio Nacional, 2007, Campinas. I Simpósio de Pesquisa em Ensino e História de Ciências da Terra / II Simpósio Nacional, 2007. CAMUS, A. Le mythe de Sisyphe. Paris: Editora Folio Essais, 1942, 87pp.

CARVALHO, M. G. P.; EDGECOMBE, G. D. Lower-Early Devonian Calmoniid trilobites from Mato Grosso, Brazil, and related species of Paraná: American Museum Novitates, n. 3022, 1991, p. 1-13.

CARVALHO, M. G. P.; MELO, J. H. G.; QUADROS, L. P. Trilobitas devonianos do Flanco Noroeste da Bacia do Paraná: Brazilian Congress of Paleontology, 10th, Rio de Janeiro, Anais, vol. 2, 1987, p. 545-565.

CLARKE, J. M. As Trilobitas da Grez de Ererê e Maecuru, Estado do Pará, Brasil. Archivos do Museu Nacional Rio de Janeiro, vol. IX, 1890,p. 1-58. 
CLARKE, J. M. Fósseis Devonianos do Paraná. Monographias do Serviço Geológico e Mineralógico do Brasil, n. 1, 1913, p. 1-353.

COOPER, M. R. A revision of the Devonian (Emsian-Eifelian) trilobita from the Bokkeveld group of South Africa. Annals of the South Africa Museum, vol. 89, n. 1, 1982, p. 1-174.

COPPER, P. Paleolatitudes in Devonian of Brazil and the Frasnian-Fammenian mass extinction. Palaeogeography, Palaeoclimatology, Palaeoecology, n. 21, 1977, p. 165-207.

ELDREDGE, N.; ORMISTON, A. R. Biogeography of Silurian and Devonian trilobites of the Malvinokaffric Realm, in Boucot, A. J. and Gray, J.: Historical biogeography, plate tectonics and the changing environment, Corvallis, Oregon University Press, 1979, p. 147-167.

ELDREDGE, N.; BRANISA, L. Calmoniid trilobites of the lower Devonian Scaphiocoelia Zone of Bolivia, with remarks on related species. Bulletin of American Museum of Natural History, no 165, 1980, p. 181290.

FIGUEIRÔA, S. F. M.. As Ciências Geológicas no Brasil: Uma História Social e Institucional, 1875-1934. São Paulo: Editora HUCITEC, 1997.

GHILARDI, R. P. Tafonomia Comparada e Paleoecologia dos Macroinvertebrados (ênfase em Trilobites), da Formação Ponta Grossa (Devoniano, Sub-bacia Apucarana), Estado do Paraná, Brasil. 2004. Tese de Doutoramento. Programa de Pós Graduação em Geologia Sedimentar, USP, São Paulo, 2004.

GHILARDI, R. P.; SIMÕES, M. G. Taphonomy and paleoautoecology of the devonian trilobites, Ponta Grossa Formation, Paraná Basin, Brazil: International Meeting on Paleoarthropodology, 1st, Ribeirão Preto, Abstracts, 2000, p. 65-66.

GHILARDI, R. P.; SIMÕES, M. G. Taphonomy of Devonian trilobites (Paraná Basin, Brazil) in a sequence stratigraphy framework- some preliminary observations: International Conference on Trilobites and their relatives, 3rd, Abstracts, p. 36. 2001.

GHILARDI, R. P.; SIMÕES, M. G. History and Development of Trilobite Research in Brazil. In: E. Landing; D.G. Mikulic; J. Kluessendorf. (Org.). Fabulous fossils--300 years of worldwide research- New York State Museum Bulletin. New York: New York Museum Press, v. 507, 2007, p. 97-104.

KATZER, F. Grundzuge der Geologie des unteren Amazonas gebiets. Berlin, Germany, 1903.

KOZLOWSKI, R. Fóssiles Devonienne du Paraná. Annales de Paleontologie, vol. VIII, 1913, p. 1-19.

LANGE, F. W. Paleontologia do Paraná, volume comemorativo do $100^{\circ}$ aniversário do Estado do Paraná, Curitiba, Brazil. 1954.

LANGE, F. W., PETRI, S. The Devonian of the Paraná Basin. Boletim Paranaense de Geociências, n. 21 and 22, 1967, p. 5-55.

MORI, A. S. Revisão sistemática dos Calmoniidae (Trilobita, Phacopida) da Formação Ponta Grossa, Devoniano, Bacia do Paraná, Brasil. Dissertação de Mestrado, Programa de Pós-Graduação em Geoquímica e Geotectônica, IGc-USP, São Paulo, 67p., 2013.

MORI, A.S.; LEME, J.M. Revisão Sistemática de Calmoniidae (Trilobita, Phacopida) da Formação Ponta Grossa (Devoniano), Bacia do Paraná, Brasil. In: I Simpósio Brasileiro de Paleontologia de Invertebrados, Bauru. Boletim de Resumos, 77. 2012.

OLIVEIRA, E. P. Fósseis Devonianos de Goyas. Boletim do Serviço Geológico e Mineralógico, SGM, n. 15, 1937, p. 2-4.

PETRI Setembrino. Contribuição ao estudo do Devoniano paranaense. Rio de Janeiro, DNPM/DGM, 125 p. 1948.

POPP, M. T. B. Revisão dos trilobitas calmoniideos e comunidades faunísticas da Formação Ponta Grossa, Devoniano do Estado do Paraná Brasil. Tese de Doutoramento. Porto Alegre, Universidade Federal do Rio Grande do Sul, 112 p., 1985.

POPP, M. T. B. Paranacaste, um novo gênero de trilobita da Formação Ponta Grossa, Bacia do Paraná. Brazilian Congress of Paleontology, 11th, Curitiba, Anais, 1989, p. 19-36. 
POPP, M. T. B.; COIMBRA, J. C.; HAUCH, A. Revisão do gênero Paracalmonia Struve, 1958 (pro Proboloides Clarke, 1913), trilobita- um ensaio em sistemática filogenética. Gaia, no 12, 1996, p. 19-32.

SIMÕES, M.G.; LEME, J.M.; SOARES, S.P. Systematics, taphonomy, and paleoecology of homalonotid trilobites (Phacopida) from the Ponta Grossa Formation. Revista Brasileira de Paleontologia, 12, 2009, p. 27-42.

SOARES, S.P. Sistemática, Tafonomia e Paleoecologia de Trilobita, Phacopida (Homalonotidae, Calmoniidae), Formação Ponta Grossa (Devoniano), Sub-bacia Apucarana, Estado do Paraná, Brasil. Dissertação de Mestrado. USP, São Paulo, SP. 1-140, 2007.

SOARES, S.P.; SIMÕES, M.G.; LEME, J.M. Metacryphaeus rotundatus, um Novo Elemento da Fauna de Trilobites Calmoniidae (Phacopida), da Formação Ponta Grossa (Devoniano), Bacia do Paraná, Brasil. Revista Geologia USP, Série Científica, 8, 2008a, p. 15-24.

SOARES, S.P.; SIMÕES, M.G.; LEME, J.M. O papel da fossilização e do intemperismo na sistemática de trilobitas Phacopida (Calmoniidae e Homalonotidae) do Devoniano da bacia do Paraná, Brasil. Revista Brasileira de Paleontologia, v. 11, 2008b, p. 59-68.

Recebido em 10/10/2013

Aceito para publicação em 02/12/2013 